\title{
Geant4 Detector Simulations for Future HEP Experiments
}

\author{
Farah Hariri*t \\ CERN, CH-1211 Geneva, Switzerland \\ E-mail: farah.haririecern.ch
}

\section{Mihaly Novak}

CERN-EP, CH-1211 Geneva, Switzerland

E-mail: mihaly.novakecern.ch

\section{Vladimir Ivanchenko}

CERN-EP, CH-1211 Geneva, Switzerland

Tomsk State University, 634050, Tomsk, Russia

E-mail: vladimir.ivantchenko@cern.ch

\section{Alberto Ribon \\ CERN-EP, CH-1211 Geneva, Switzerland \\ E-mail: alberto.ribon@cern.ch}

The experimental programmes planned for the next decade are driving developments in the simulation domain: these include the High Luminosity LHC project (HL-LHC), neutrino experiments (LBNF/DUNE), and studies towards future facilities such as Linear Collider (ILC/CLIC) and Future Circular Collider (FCC). The complex detectors of the future, with different module- or cell-level shapes, finer segmentation, and novel materials and detection techniques, require additional features in geometry tools and bring new demands on physics coverage and accuracy within the constraints of the available computing resources. In order to achieve the desired precision in physics measurements, while avoiding that simulation dominates the systematic uncertainties, more accurate simulations and larger Monte Carlo samples will be needed. Therefore, this sets the challenge to develop more accurate models of physics interactions with affordable computing time [1]. The widely used detector simulation toolkit Geant4 [2,3] is at the core of simulation in almost every HEP experiment. In this paper, we will discuss the status of Geant4 in the context of detector R\&D for present and future facilities. We highlight, in particular, the need to review some of the physics models' assumptions, approximations and limitations in order to increase precision, and to extend the validity of models up to future circular collider energies of the order of $100 \mathrm{TeV}$. Examples of recent improvements in electromagnetic models will be presented in detail.

The 39th International Conference on High Energy Physics (ICHEP2018)

4-11 July, 2018

Seoul, Korea

\footnotetext{
* Speaker.

${ }^{\dagger}$ On behalf of the Geant 4 collaboration
} 


\section{Electromagnetic physics for High-Luminosity LHC and future colliders}

Electromagnetic (EM) transport simulation is challenging as it occupies a significant part of the computing resources used in full detector simulation. More experimental data in the coming decade implies higher statistics. We therefore need large and accurate simulation samples, which require both model accuracy and speed. Geant 4 physics libraries provide simulation for a wide variety of applications in HEP, space science, medical physics and other scientific domains. To be ready for High-Luminosity and future colliders simulations, our strategy is to improve shower description by refining the physics models at the microscopic level, aiming at improvements at the macroscopic level. In electromagnetic physics, substantial progress has been made by revising the theoretical description of models such as multiple scattering, bremsstrahlung and pair-production. These models have been rewritten from scratch and new implementations are available in Geant 4 10.4. One of the most complicated processes that needs to be described accurately is the multiple scattering of charged particles in matter. Currently the Urban parametrised multiple scattering model [4] is used by default in Geant 4 for $e^{-}$below $100 \mathrm{MeV}$. The newly proposed model is the fully theory-based Goudsmit-Saunderson (GS) model for electron/positron multiple scattering with the distinct feature of not being sensitive to geometry nor to step-size [6, 5]. In Figure 1 we show the angular distribution of a $15.7 \mathrm{MeV} e^{-}$transmitted through a thin gold layer. We compare the default Urban model (blue solid line) and the theory-based GS model (red solid line) to experimental data (black dots). At the bottom of the graph we show the deviation in percent of both models with respect to data. The deviation is more than $20 \%$ for the default Urban model, whereas it is less than $5 \%$ for the new theory-based model in red. In conclusion, this is a major improvement of the multiple scattering model now present in Geant4 that will likely improve accuracy for various use-cases including electromagnetic shower shape. We also improved the

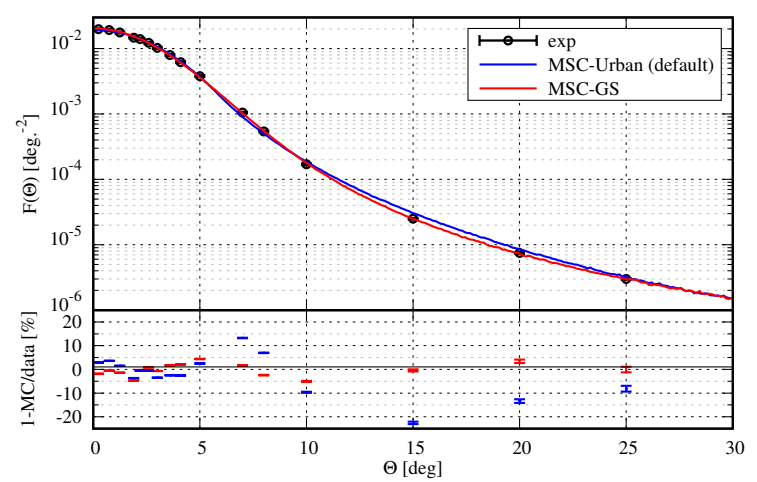

Figure 1: The new Goudsmit-Saunderson theoretical model of multiple scattering in red solid line vs experimental data from A.O. Hanson et al., Phy. Rev. 84 (4)(1951), 634.

models at high energies. Because ultra-relativistic electromagnetic interactions involve very small longitudinal momentum transfers, the reactions occur gradually over the formation length. Due to multiple scattering along this length, electromagnetic radiation is supressed and the effect is called the Landau-Pomeranchuk-Migdal (LPM) effect. We first considered the bremsstrahlung process. Revising the theoretical description of the LPM effect showed inconsistencies in the calculation of the LPM suppression variables in Geant4 10.2 and earlier versions. In Figure 2 (left) we plot 
the differential cross-section of the emitted $\gamma$ for a $25 \mathrm{GeV} e^{-}$on a thin lead target and compare the old bremsstrahlung model (blue dashed line) to the revised one with a new treatment of LPM suppression (red solid line) with respect to experimental data (black dots). The new bremsstrahlung model shows excellent agreement with the available data. These improvements were made without introducing any speed penalty. Bremsstrahlung and pair creation are two of the most common high
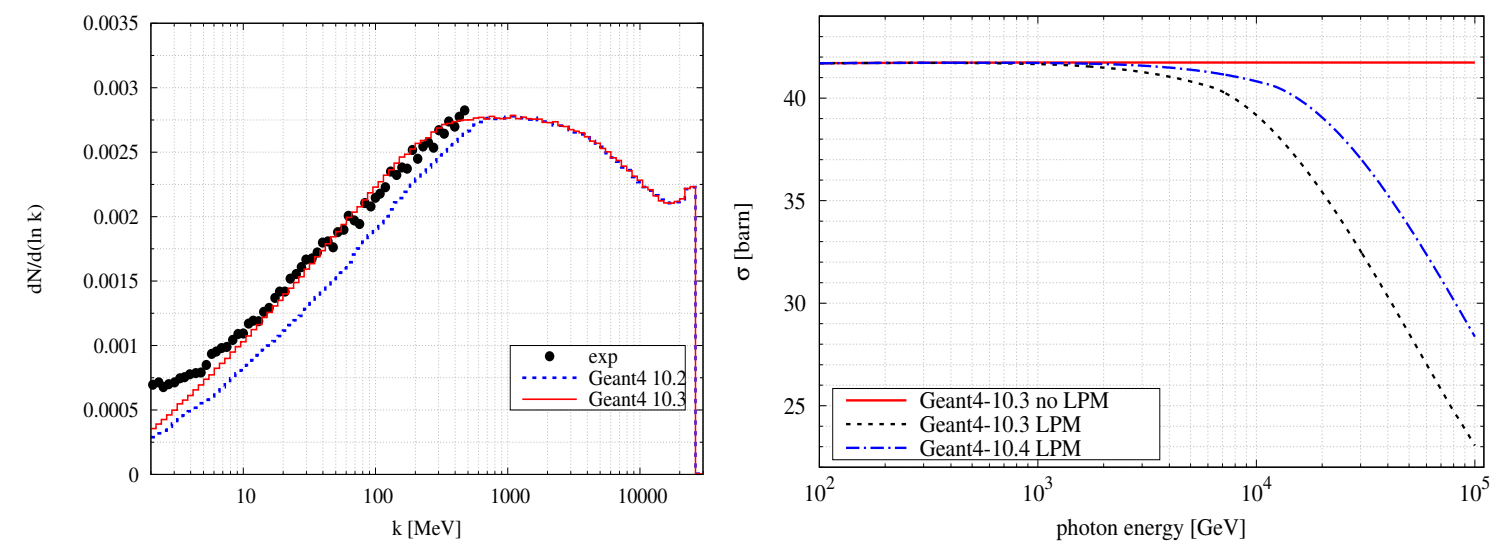

Figure 2: The revised bremsstrahlung model (left) in agreement with experimental data from P.L. Anthony et al., Phy. Rev.D. 56 (1997), 1373. The pair-production model (right) extended up to FCC-hh energies

energy electromagnetic processes, related by cross-symmetry of their Feynmann diagrams. So pair creation is also sensitive to its environment and, not surprisingly, has an LPM suppression similar to the bremsstrahlung case. However, there is one difference with respect to bremsstralung, which is that the multiple scattering now applies to two separate particles. The model describing the pairproduction process has been revised. A new model is proposed with LPM suppression functions corrected according to quantum mechanical calculations. Since there is no good data on the LPM effect in pair production, we only validate the revised bremsstrahlung model with data from SLAC E-146 and the CERN experiments and trust crossing symmetry. In Figure 2 (right), we consider gamma conversion in lead and we plot the integrated cross-section of gamma conversion as a function of the photon energy in $\mathrm{GeV}$. In red, we plot a reference case, the model without LPM to show the importance of this effect at high energies; in a black dashed line, the old Geant 4 model and in blue the new one. The old model is over-suppressing $\gamma$ conversion i.e., the LPM effect was overestimated. At very high energy the improved pair production model shows a significant difference in the differential cross-section and is expected to have an impact on energetic gammas of importance for FCC-hh.

\section{Hadronic physics future requirements}

The most important application of hadronic physics in HEP is the simulation of hadronic showers, which is one of the main components for the simulation of jets. The precision of the simulation of hadronic showers is in the range $10 \%-20 \%$ whereas that of electromagnetic showers is of the order of the $1 \%$ level. It is not possible for a single model to describe all the physics encountered in a hadronic physics simulation due to the large energy range that needs to be covered and the simplified approximations that are used in hadronic models to overcome the difficulty of 
solving the full theory (QCD). There are three major components of hadronic physics models from high to low energy: string models, intranuclear cascade models and low energy nuclear models. For high luminosity there will be significantly more data. Depending on the type of analysis, one can be more demanding on the simulation accuracy. For instance, typical Beyond Standard Model (BSM) searches are using fast simulation. However, electroweak and QCD studies sometimes require highly precise detector simulation (20-30 TeV jets for FCC-hh). The biggest amount of work to be performed in hadronic physics to match the requirements of future experiments is on string models. We need to extend the validity of string models to multi-TeV energies, necessary for accelerator applications, FCC-hh detector simulations studies and high-energy cosmic rays. We also need to include the treatment of hadronic interactions for charm and bottom hadrons.

\section{Perspectives in simulation for future HEP experiments}

Theoretical review of all electromagnetic models, including those of hadrons and ions, is a high priority both for HL-LHC and even more importantly for FCC-hh studies. In this paper we discussed three major improvements now available in Geant4 10.4, but there is a substantial amount of work still needed for precise modeling of showers for future HEP experiments. Avenues that should be investigated include other channels that become important at very high energies, such as triplet production taking into account nuclear recoil effects, nuclear size effects as well as $\gamma$ conversion to muon and hadron pairs. Energy fluctuation models should be developed based on theory, not just on parametrisation. Precision is crucial for High-Luminosity LHC to limit the systematic error and extension of the model validity is necessary to reach Future Circular Collider and cosmic ray energies. To cope with these future experimental needs, our strategy consists of going back to the theoretical underpinnings and reviewing major physcis models: multiple scattering, bremsstrahlung and pair-production. Revising the theory, assumptions, approximations and implementations of the latter models from scratch show improvements (available in Geant4 10.4) that will affect showers without penalising performance and possibly reducing the discrepancies reported by experiments. The readiness for future HEP experiments requires first getting the best physics accuracy and better performance in full simulation, by getting as close as possible to using true QED and QCD predictions. Whenever possible, we prefer using theory-based approaches to data-driven approaches. The second challenge is to make the software as fast as possible, thus permitting the production of more simulation events with the available computing resources.

\section{References}

[1] HEP Software Foundation Community White Paper (2018), arXiv preprint arXiv:1803.04165.

[2] S. Agostinelli, et al., GEANT4 - a simulation toolkit, NIMA 506 (3)(2003) 250-303.

[3] J. Allison, et al., Recent developments in Geant4, NIMA 835 (2016) 186-225.

[4] L. Urban, Multiple scattering model in Geant4, CERN-OPEN-2002-070.

[5] S. Incerti, V. Ivanchenko, M. Novak, Recent progress of Geant4 electromagnetic physics for calorimeter simulation, Journal of Instrumentation, 13 (02) (2018) C02054.

[6] S. Goudsmit, J.L. Saunderson. Multiple scattering of electrons, Physical Review, 57(1) (1940), 24. 